\title{
Differential role of spermine and thermospermine in Arabidopsis thaliana in response to abiotic stresses
}

\author{
Afsana Haque, Nur-E-Ferdousy, Sharif Ar Raffi and G H M Sagor \\ Department of Genetics \& Plant Breeding, Bangladesh Agricultural University, Mymensingh-2202, Bangladesh
}

\begin{tabular}{|c|c|}
\hline ARTICLE INFO & Abstract \\
\hline $\begin{array}{l}\text { Article history: } \\
\text { Received: } 12 \text { July } 2018 \\
\text { Accepted: } 08 \text { August } 2018\end{array}$ & \multirow{3}{*}{$\begin{array}{l}\text { Polyamines (PA) are small organic polycations found in all living organisms. Putrescine (Put), spermidine } \\
\text { (Spd), spermine (Spm) and thermospermine (T-Spm) are the most abundant PAs in plants. Of the } \\
\text { polyamines (PAs) plant contains at least two types of tetraamines; spermine (Spm) and thermospermine } \\
\text { (T-Spm) and they seem to have different functions in plants. The experiment was conducted to eludicate } \\
\text { the stress responsive roles of Spm and T-Spm using wild ecotype (Col-0) and three different knockout } \\
\text { mutants (spms, acl5 and spms/acl5) of Arabidopsis, differing in the amount of spermine and } \\
\text { thermospermine content. First exogenous pretreatment of both tetraamines showed their different effects } \\
\text { on plant phenotype including root and shoot length, cholorophyll content both under normal and salt } \\
\text { stressed condition. The effect of exogenous T-Spm was more effective than Spm in plant growth and } \\
\text { stress tolerance. The mutants varying in endogenous tetraamines content were also found to be different in } \\
\text { response to stress (salt, osmotic and ionic) condition. Growth inhibition of spms mutant seedlings was } \\
\text { minimum in compare to other mutants and wild type, probably due to higher amount of T-Spm content. } \\
\text { Further to differentiate the tissue specific expression of tetramines, histochemical GUS assay was } \\
\text { performed using SPMS and ACL5 promoter:: GUS transgenic plants and the results indicate that the two } \\
\text { tetraamines; spermine and thermospermine may play some different and specific role in respect to tissue } \\
\text { specificity and stress responses. }\end{array}$} \\
\hline $\begin{array}{l}\text { Polyamines, Spermine and } \\
\text { Thermospermine, Abiotic Stress }\end{array}$ & \\
\hline & \\
\hline
\end{tabular}

\section{Introduction}

Polyamines (PA) are positively charged low molecular weight aliphatic molecule having two or more amino groups, found in almost all living organisms (Kusano et al., 2007). Due to polycationic nature, they have high affinity to different anionic cellular components such as DNA, RNA and protein. Major polyamines in plants are diamine putrescine (Put), triamine spermidine (Spd), tetraamine spermine (Spm) and thermospermine (TSpm). (Kusano et al., 2008; Fuell et al., 2010; Tiburcio et al., 2014). Polyamine plays major role in plant growth, development as well as cell division. They are involved not only in fundamental cellular processes but also in various environmental stresses (Alcazar et al., 2006; Bagni et al., 2006; Kusano et al., 2008).

In plants, PA biosynthesis starts with the formation of Put, which is synthesized from ornithine by ornithine decarboxylase. Putrescine is also synthesized from arginine via agmatine by three sequential reactions catalyzed by arginine decarboxylase (ADC), agmatine iminohydrolase (AIH), and $N$-carbamoylputrescine aminohydrolase (CPA), respectively. Then, Put is converted to Spd and $\mathrm{Spm}$ via two sequential aminopropyl transferase reactions catalyzed by Spd synthase (SPDS) and Spm synthase (SPMS), respectively. In both cases, an aminopropyl residue is transferred from the decarboxylated $S$ adenosylmethionine which is synthesized by $S$ adenosylmethionine decarboxylase (SAMDC) (see review, Kusano, et al., 2008). The genome of the model plant Arabidopsis thaliana does not contain an ornithine decarboxylase (ODC) gene (Hanfrey et al., 2001). In this organism, it had been believed that two genes, Acaulis 5 (ACL5) and Spermine synthase (SPMS), encode SPMS. However, Knott et al. (2007) reported that the bacterially expressed recombinant ACL5 protein catalyzes the conversion of Spd to T-Spm, but not to Spm, indicating that ACL5 encodes T-Spm synthase. The above discussion indicates that Arabidopsis plants contain four major PAs including T-Spm. T-Spm was first discovered in the thermophilic bacterium Thermus thermophilus (Oshima, 1979) and then found in several plants including Pisum sativum, Vicia sativa and Malus sylvestris (Hamana et al., 1991; Kitashiba et al., 2005). Recently, Naka et al. (2009) detected T-Spm in Nicotiana tabacum, Lycopersicon esculentum and Oryza sativa. The gathered evidence suggests that T-Spm seems to be ubiquitously present in plants. As all the genes involved in PA biosynthetic pathway identified in A. thaliana and thus the genetic analyses of the loss/gain-of-function types became possible. With such analyses, it has been revealed that Put and Spd are essential for normal growth of Arabidopsis, while Spm and T-Spm are not (Alcazar et al., 2006). Arabidopsis (spms/acl5) mutant plant that cannot produce spermine, was hypersensitive to drought and salinity and the hypersensitive phenotype was mitigated by application of exogenous spermine and the same mutant also showed a phenotype resembling $\mathrm{Ca}^{2+}$-deficiency (Yamaguchi et al., 2006). Arabidopsis plants overexpressing Cucurbita ficilolia Spd synthase gene became tolerant to multiple stresses such as chilling, 
freezing, salinity, drought and paraquat-induced oxidative stress (Kasukabe et al., 2004). Similarly, several other plant species also shown that polyamine accumulation occurs in response to several adverse environmental conditions, including salinity, drought, chilling, heat, hypoxia, ozone, UV-B and UV-C, heavy metal toxicity, mechanical wounding and herbicide treatment (Alcázar et al., 2006; Groppa et al., 2008). $\mathrm{Spm}$, one of the tetraamines, has a signalling function to induce a defense reaction against avirulent viral pathogen. Similar evidence was obtained in the combination of $A$. thaliana carrying the leucine rich repeat type resistant gene $R C Y 1$ and the yellow strain of cucumber mosaic virus (CMV) (Mitsuya et al., 2009). Recently, proven that another tetraamine, T-Spm also has an inducing activity of the expression of genes involving in the same Spm-signal pathway and of the genes involving in CMV-triggered hypersensitive response (HR) and consistent with the gene-inducing activity, TSpm can also represses the CMV multiplication as efficiently as Spm does (Sagor et al., 2012). In this study, we examined the roles of both exogenous and endogenous Spm and T-Spm in response to abiotic stresses and also their tissue specific expression in Arabidopsis thaliana.

\section{Materials and Methods}

Experimental site, plant materials and growth condition

The experiment was conducted at the Tissue Culture Laboratory and Plant Molecular Genetics Laboratory, Department of Genetics and Plant Breeding, Bangladesh Agricultural University, Mymensingh. The plant materials consists of three different Arabidopsis mutants namely spms, acl5 and spms/acl5 including wild type Col-0. The list of the plant materials and their characteristics has been shown in Table 1. Seeds of all mutants and wild type were surface sterilized with $70 \%$ ethanol for $1 \mathrm{~min}$ and $1 \%$ sodium hypochloride plus $0.1 \%$ Tween-20 for $15 \mathrm{~min}$, followed by extensive washing with sterile distilled water. After sterilization seeds were placed on half-strength MS (Murashige and Skoog, 1962) 1.5\% agar plates ( $\mathrm{pH} 5.6)$ containing $1 \%$ sucrose and B5 vitamin (MP Biomedicals, Cat\#2625149). The plants were grown at $22{ }^{\circ} \mathrm{C}$ under a 14h light /10h dark photocycle.

Table 1. Plant materials, characteristics and source of the material

\begin{tabular}{lll}
\hline \multicolumn{1}{c}{$\begin{array}{c}\text { Plant } \\
\text { Material }\end{array}$} & \multicolumn{1}{c}{ Special characteristics } & \multicolumn{1}{c}{ Source } \\
\hline $\begin{array}{l}\text { Col-0 } \\
\text { spms }\end{array}$ & $\begin{array}{l}\text { Polyamines are in normal level } \\
\text { No Spm, slightly increased level of } \\
\text { T-Spm }\end{array}$ & $\begin{array}{l}\text { Sagor } \text { et al., 2013 } \\
\text { Sagor } \text { et al., 2013 }\end{array}$ \\
acl5 & $\begin{array}{l}\text { No T-Spm, slightly increased level } \\
\text { of Spm }\end{array}$ & Sagor et al., 2013 \\
spms/acl5 & $\begin{array}{l}\text { No Spm and T-Spm } \\
\text { SPMS } \\
\text { pro::GUS }\end{array}$ & Polyamines are in normal level \\
$\begin{array}{l}\text { ACL5 } \\
\text { pro::GUS }\end{array}$ & Polyamines are in normal level Sal., 2013 \\
\hline
\end{tabular}

\section{Growth response to stress condition}

Sterilized Arabidopsis seeds were grown on 1/2 MS agar plates containing different concentration of $\mathrm{NaCl}(0,25$, 50, $100 \mathrm{mM}$ ); Polyethylene glycol (PEG-6000) $(0 \%$, $10 \%, 15 \%, 20 \%) ; \mathrm{CaCl}_{2}(0,6,12,50 \mathrm{mM})$ and $\mathrm{KCl}(0$, $50 \mathrm{mM})$. The plates were placed at a vertical position with an 85 degree angle and incubated at $22^{\circ} \mathrm{C}$ for 14 days. $\mathrm{NaCl}, \mathrm{PEG}, \mathrm{CaCl}_{2}$ and $\mathrm{KCl}$ were used to induce salt, osmotic and ionic stress, respectively.

\section{Pretreatment with Spm and T-Spm solution}

Ten days old seedlings of Col-0 were carefully detached from the MS agar plate, rinsed with distilled water, placed the root in wet filter paper containing $500 \mu \mathrm{M}$ Spm and $100 \mu \mathrm{M}$ T-Spm solution for $24 \mathrm{hrs}$. Then, the plants were carefully transferred to 0,100 and $200 \mathrm{mM}$ $\mathrm{NaCl}$ containing $\mathrm{MS}$ media and shifted to tissue culture room at $22^{\circ} \mathrm{C}$.

\section{Data collection and analysis}

Root length (cm): Length of root was measured in centimeter $(\mathrm{cm})$ from the base to the tip of the roots by a measuring scale at 14 days after initiation.

Shoot length (cm): The shoot length was measured with the help of centimeter scale by placing the plants straightly on glass slide from the starting part of main stem to tip of the upper leaf.

Leaf area: Leaf area was measured under digital optical microscope (Kenis).

Leaf Chlorophyll content [CC] (SPAD unit): Chlorophyll content was measured from one leaves per replication using the SPAD (Minolta-502).

Each experiment was repeated three times and the mean values of collected numerical data were analyzed using Microsoft Excel-2007 software.

\section{Histochemical GUS assay}

The histochemical localization of GUS activity was assayed according to the method of Jefferson, 1987; Sagor et al., 2011). Samples were collected and incubated with GUS staining solution $[0.5 \mathrm{mg} / \mathrm{ml} \mathrm{X-Glu}$ (5-bromo-4- chloro-3-indolyl b-D-glucuronide), $10 \mathrm{mM}$ phosphate buffer, $\mathrm{pH} 7.0,0.5 \mathrm{mM}$ potassium ferricyanide, $0.5 \mathrm{mM}$ potassium ferrocyanide, $0.1 \%$ Triton X-100, $10 \mathrm{mM}$ EDTA] in the dark at $37{ }^{0} \mathrm{C}$ for overnight. After incubation, stained plant cells were cleared by $70 \%$ ethanol to remove the chlorophyll. Samples were taken on light plate and photographed using digital camera (Canon R250, Japan).

\section{Chemicals}

Spm were purchased from Tokyo Kasei Co. Ltd. (Tokyo, Japan). T-Spm was chemically synthesized (Niitsu and Samejima, 1986). All other chemicals were obtained at the analytical grade from Sigma-Aldrich Corp (St. Louis, MO, USA), Wako Pure Chemical Industries Ltd. (Osaka, Japan) and Nacalai-Tesque Ltd. (Kyoto, Japan). 


\section{Results}

Effect of exogenous tetraamines in response to salt stress

In order to notice the effect of exogenous tetraamines, the wild type plants were grown on normal MS media, treated with water (Mock), $500 \mu \mathrm{M} \mathrm{Spm}$ and $100 \mu \mathrm{M}$ T-

A.

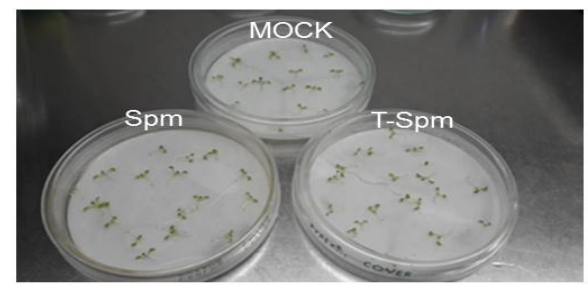

B.

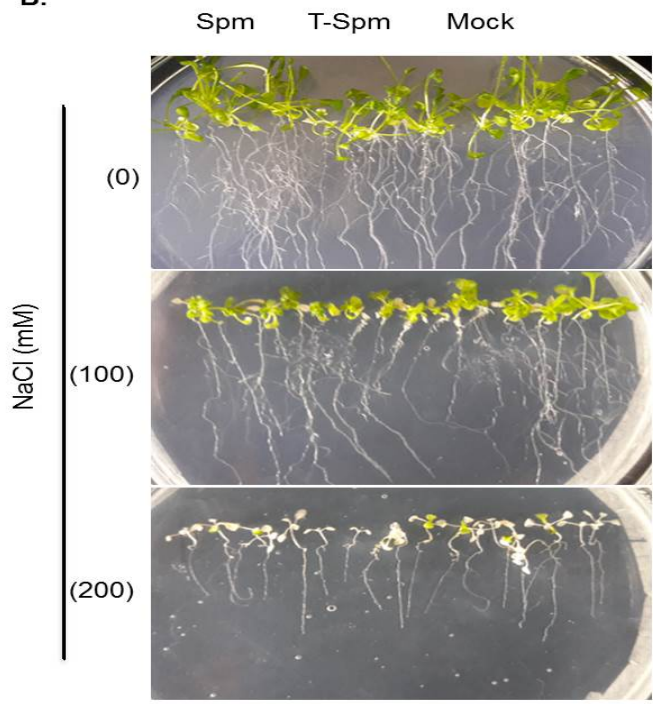

Spm for $24 \mathrm{hrs}$ and then transferred to with or without $\mathrm{NaCl}$ containing media. Seven days after incubation, TSpm pretreated plant showed higher root length, shoot length and chlorophyll content compare to Spm and mock one (Fig. 1A, 1B, 1C) both in control and salt stressed conditions.

c.
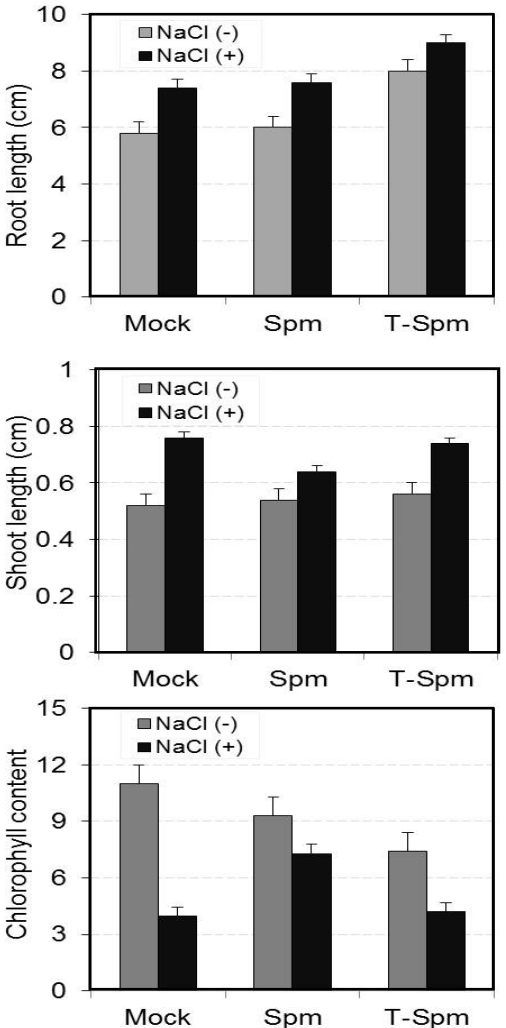

Fig. 1. Effect of exogenously applied Spm and T-Spm to protect Arabidopsis seedlings from salt induced damage. A. Pretreatment of ten days old Arabidopsis seedlings with $0.5 \mathrm{mM} \mathrm{Spm}$ and $0.1 \mathrm{mM}$ T-Spm for $24 \mathrm{hrs}$. B. Pretreated plants were transferred to 0,100 and $200 \mathrm{mM} \mathrm{NaCl}$ containing MS media and incubated at $22^{\circ} \mathrm{C}$ for another 5 days and the photograph was taken. C. Root lengh, shoot length and chlorophyll content in wild type and different tetraamine mutants. Mean values $\pm \mathrm{SE}$ were shown using three time repetitions.

\section{Sensitivity of tetraamines deficient mutant to salt stress}

The growth response of three mutants namely spms, acl5, acl5/spms and wild type (Col-0) of Arabidopsis thaliana were tested under both control and salt stress condition created by using different concentration of $\mathrm{NaCl}(0,25,50$ and $100 \mathrm{mM} \mathrm{NaCl}$ respectively). Both the wild type and mutant plants were tall, healthy, and vigorous with juvenile leaves, petiole and stems as well as well-established root system at normal physiological condition, but in case of salt stress condition Col-0 showed higher sensitivity whereas spms showed the tolerance (Fig. 2). The root length and shoot length were decreased with the increasing concentration of $\mathrm{NaCl}$. At strong salt stress condition, root and shoot length were maximum in spms mutant and minimum in spms/acl5 mutant (Fig. 2; Table 2). Leaf area and total chlorophyll content also showed similar results that spms performed the best among the wild type and mutant plants (Table 2).

\section{Growth response of Arabidopsis to osmotic stress}

Next, the growth response of wild type and spms, acl5, spms/acl5 mutants of Arabidopsis thaliana were tested under osmotic stress condition created by using PEG solution. The wild type and mutant lines (spms, acl5, acl5/spms) showed varying levels of tolerance under osmotic stress. The growth of plants becomes severely affected at 20\% PEG (Fig. 3). Leaves became small and curly, petiole became short and curved, and plant height became also reduced with the increase of PEG level (Fig. 3). Col-0 was highly sensitive to PEG at $20 \%$ level as root length and shoot length were very short and spms plants were tolerant (Table 3). Other mutant plants showed similar type of sensitivity (Fig. 3). Total leaf area and chlorophyll content were also found to be maximum in spms mutant whereas minimum in spms/acl5 plants at high level of osmotic (20\%) stress (Table 3). 


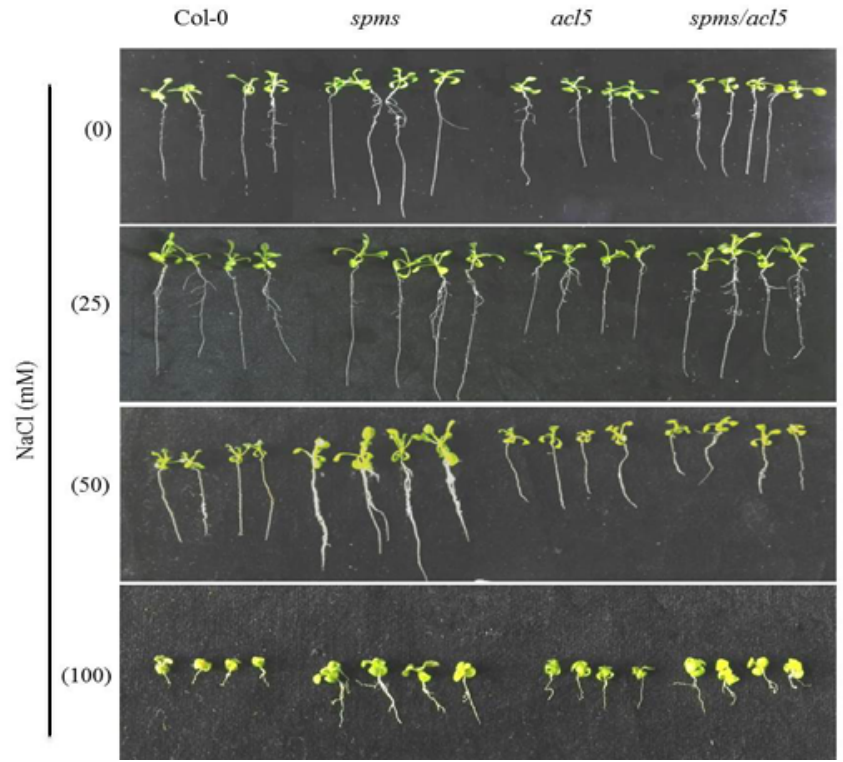

Fig. 2. Growth response of wild type and tetraamine deficient mutant in response to salt stress. Seeds of wild type, spms, acl5 and spms/acl5 mutants were sown on $0,25,50$ and $100 \mathrm{mM} \mathrm{NaCl}$ containing MS media, then kept on $4^{0} \mathrm{C}$ for two days and then transferred to growth room at $22^{\circ} \mathrm{C}$. Photographs were taken after 14 days of incubation

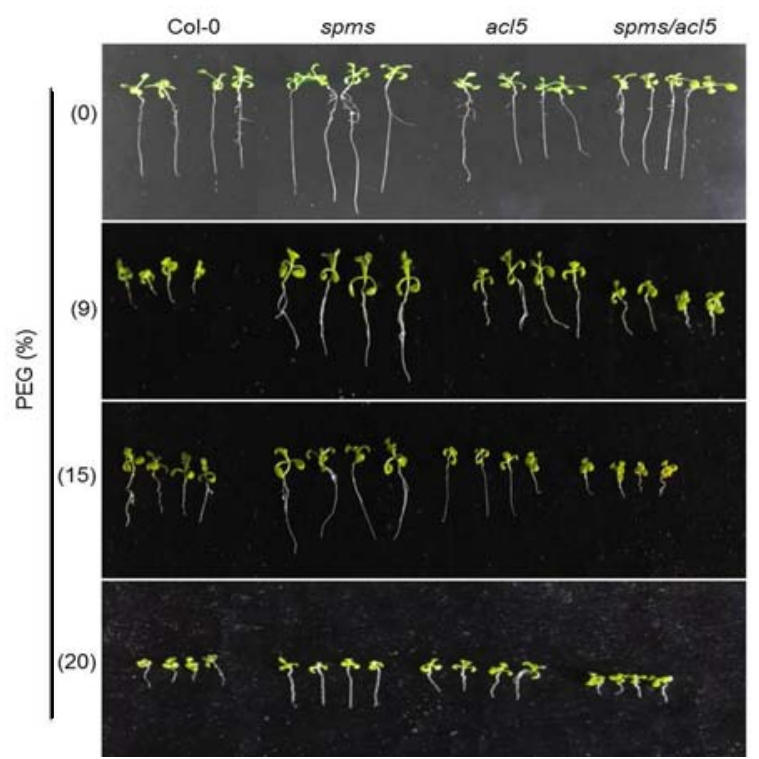

Fig. 3. Phenotypic performance of wild type and tetraamine deficient mutant to osmotic stress. Seeds of wild type, spms, acl5 and spms/acl5 mutants were sown on $0 \%$, $10 \%, 15 \%$ and $20 \%$ PEG containing MS media, then kept on $4^{0} \mathrm{C}$ for two days and then transferred to growth room at $22^{\circ} \mathrm{C}$. Photographs were taken after 14 days of incubation

Table 2. Phenotypic characterization of Col-0, spms, ac15 and spms/ac15 in response to salt stress

\begin{tabular}{|c|c|c|c|c|c|c|c|c|c|c|c|c|c|c|c|c|}
\hline \multirow{3}{*}{$\begin{array}{l}\text { Plant } \\
\text { materials }\end{array}$} & \multicolumn{16}{|c|}{$\mathrm{NaCl}(\mathrm{mM})$} \\
\hline & \multicolumn{4}{|c|}{$\mathrm{RL}(\mathrm{cm})$} & \multicolumn{4}{|c|}{ SL $(\mathrm{cm})$} & \multicolumn{4}{|c|}{$\mathrm{LA}\left(\mathrm{mm}^{2}\right)$} & \multicolumn{4}{|c|}{ CC (unit) } \\
\hline & 0 & 25 & 50 & 100 & 0 & 25 & 50 & 100 & 0 & 25 & 50 & 100 & 0 & 25 & 50 & 100 \\
\hline Col-0 & 2.3 & 2.7 & 2.1 & 0.6 & 1.2 & 1.0 & 0.4 & 0.1 & 6.0 & 5.4 & 4.1 & 2.5 & 5.0 & 4.1 & 2.9 & 0.6 \\
\hline spms & 3.6 & 3.6 & 3.0 & 1.2 & 1.5 & 1.1 & 0.5 & 0.2 & 6.5 & 5.8 & 4.5 & 3.2 & 6.9 & 4.7 & 3.5 & 0.9 \\
\hline ac15 & 2.6 & 2.4 & 1.9 & 0.4 & 1.0 & 0.7 & 0.2 & 0.1 & 5.5 & 5.0 & 3.7 & 2.5 & 5.2 & 3.8 & 2.6 & 0.4 \\
\hline spms/ac15 & 2.7 & 2.2 & 1.7 & 0.6 & 1.0 & 0.6 & 0.2 & 0.1 & 5.4 & 5.4 & 3.6 & 2.6 & 5.4 & 3.1 & 2.6 & 0.3 \\
\hline
\end{tabular}

Foot note: Root length (RL); Shoot length (SL); Leaf area (LA) and Chlorophyll content (CC)

Table 3. Phenotypic characterization of Col-0, spms, ac15 and spms/ac15 in response to osmotic stress

\begin{tabular}{|c|c|c|c|c|c|c|c|c|c|c|c|c|c|c|c|c|}
\hline \multirow{3}{*}{$\begin{array}{l}\text { Plant } \\
\text { materials }\end{array}$} & \multicolumn{16}{|c|}{$\mathrm{NaCl}(\mathrm{mM})$} \\
\hline & \multicolumn{4}{|c|}{$\mathrm{RL}(\mathrm{cm})$} & \multicolumn{4}{|c|}{$\mathrm{SL}(\mathrm{cm})$} & \multicolumn{4}{|c|}{$\mathrm{LA}\left(\mathrm{mm}^{2}\right)$} & \multicolumn{4}{|c|}{ CC (unit) } \\
\hline & 0 & 10 & 15 & 20 & 0 & 10 & 15 & 20 & 0 & 10 & 15 & 20 & 0 & 10 & 15 & 20 \\
\hline Col-0 & 2.3 & 1.1 & 1.0 & 0.4 & 1.1 & 0.8 & 0.3 & 0.2 & 6.0 & 2.5 & 2.5 & 1.8 & 5.6 & 2.5 & 2.1 & 1.9 \\
\hline spms & 3.5 & 1.5 & 1.3 & 0.8 & 1.5 & 1.2 & 0.6 & 0.3 & 6.5 & 3.8 & 3.6 & 2.3 & 7.0 & 3.1 & 2.9 & 2.0 \\
\hline ac15 & 2.6 & 0.3 & 0.6 & 0.4 & 1.0 & 0.7 & 0.2 & 0.1 & 5.6 & 3.0 & 2.9 & 2.2 & 5.2 & 2.1 & 1.9 & 0.9 \\
\hline spms/ac15 & 2.7 & 0.2 & 0.5 & 0.3 & 1.0 & 0.5 & 0.3 & 0.1 & 5.4 & 2.3 & 2.1 & 1.9 & 5.4 & 2.1 & 1.7 & 0.7 \\
\hline
\end{tabular}

*Root length (RL); Shoot length (SL); Leaf area (LA); and Chlorophyll content (CC)

Effect of ionic imbalance on the growth response of Arabidopsis

Finally the role of tetraamines in response to ionic imbalance condition was evaluated using wild type and tetraamine deficient mutants using $\mathrm{CaCl}_{2}$ and $\mathrm{KCl}$ as inducers. The growth of all the plants was inhibited but the rate of decrease was minimum in spms mutant whereas other three showed almost same degree and level (Fig. 4). Root and shoot length, leaf area, chlorophyll content all were maximum in spms mutant compared to others indicating tolerance to ionic imbalance conditions (Fig. 4; Table 4). 


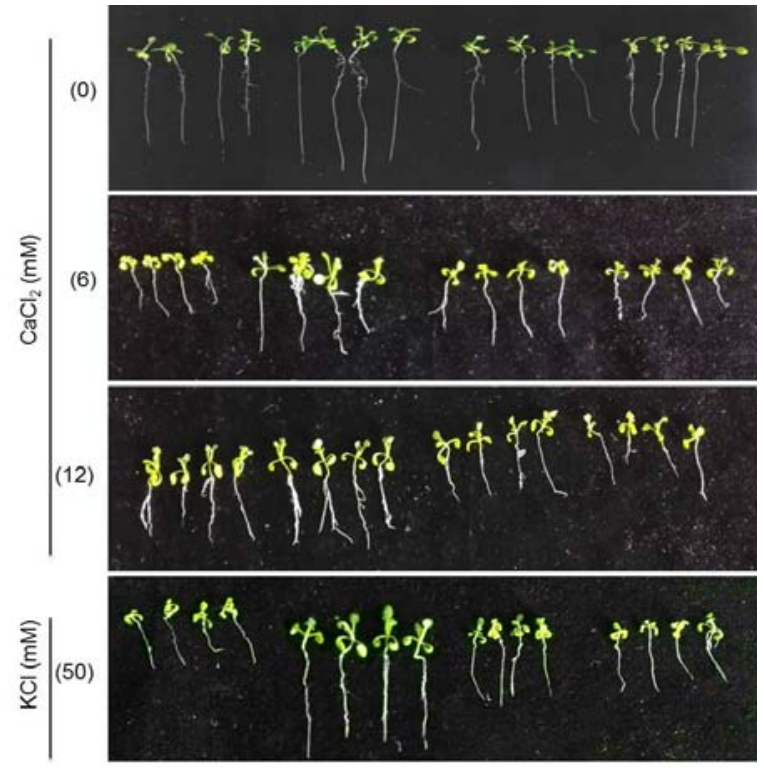

Fig. 4. Effect of ionic imbalance on growth response of Arabidopsis thaliana. Seeds of wild type, spms, acl5 and spms/acl5 mutants were sown on 6 and $12 \mathrm{mM} \mathrm{CaCl}$ and $50 \mathrm{mM} \mathrm{KCL}$ containing MS media, were kept on $4^{0} \mathrm{C}$ for two days and then transferred to growth room at $22^{\circ} \mathrm{C}$. Photographs were taken after 14 days of incubation

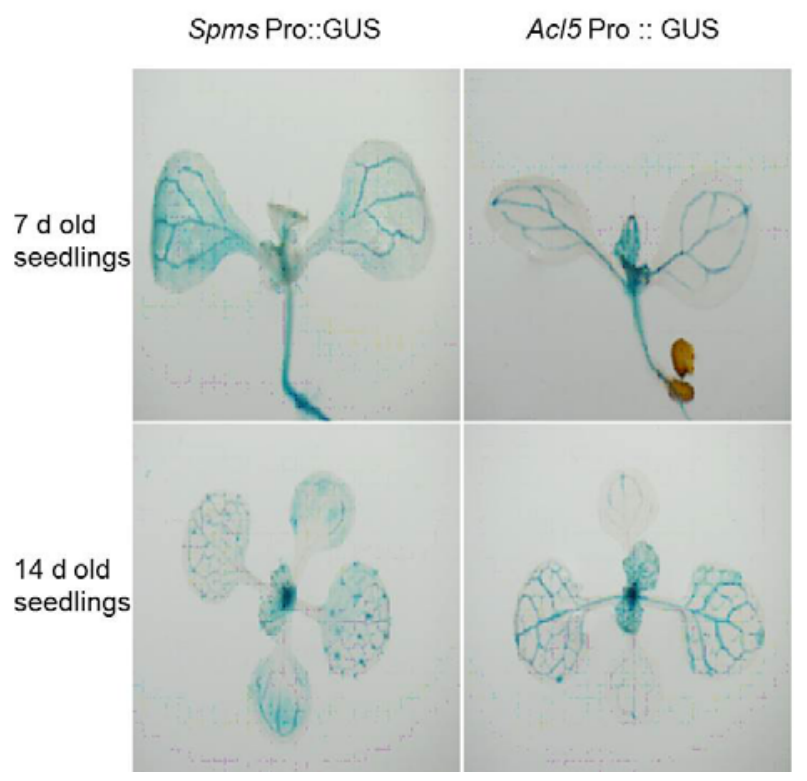

Fig. 5. Expression patterns of Spms- and Acl5-promoter driven beta-glucuronidase reporter gene (GUS) in transgenic plants. Degree of blue staining reflects the promoter activity

Table 4. Phenotypic characterization of Col-0, spms, ac15 and spms/ac15 in response to ionic stress

\begin{tabular}{|c|c|c|c|c|c|c|c|c|c|c|c|c|c|c|c|c|c|c|c|c|}
\hline \multirow{3}{*}{$\begin{array}{l}\text { Plant } \\
\text { materials }\end{array}$} & \multicolumn{12}{|c|}{$\mathrm{CaCl}_{2}(\mathrm{mM})$} & \multicolumn{8}{|c|}{$\mathrm{KCl}(\mathrm{mM})$} \\
\hline & \multicolumn{3}{|c|}{ RL (cm) } & \multicolumn{3}{|c|}{$\mathrm{SL}(\mathrm{cm})$} & \multicolumn{3}{|c|}{$\mathrm{LA}\left(\mathrm{mm}^{2}\right)$} & \multicolumn{3}{|c|}{$\mathrm{CC}$ (unit) } & \multicolumn{2}{|c|}{$\mathrm{RL}(\mathrm{cm})$} & \multicolumn{2}{|c|}{$\mathrm{SL}(\mathrm{cm})$} & \multicolumn{2}{|c|}{$\mathrm{LA}\left(\mathrm{mm}^{2}\right)$} & \multicolumn{2}{|c|}{$\mathrm{CC}$ (unit) } \\
\hline & 0 & 6 & 12 & 0 & 6 & 12 & 0 & 6 & 12 & 0 & 6 & 12 & 0 & 50 & 0 & 50 & 0 & 50 & 0 & 50 \\
\hline$\overline{\mathrm{Col}-0}$ & 2.3 & 1.0 & 1. & 1.2 & 0.5 & 0 & 6.0 & 2.3 & 2.2 & 5.6 & 2.1 & 1. & 2.3 & 0.6 & 1.2 & 0.1 & 6.0 & 9 & 5.6 & .9 \\
\hline spms & 3.5 & 1. & 1. & 1. & 0.8 & 0.5 & 6.5 & 3 & 2.9 & 6.9 & 2.9 & 2.1 & 3.5 & 0.8 & 1.5 & 0.2 & 6.5 & 2.5 & 6.9 & 1.3 \\
\hline ac15 & 2.6 & 0.6 & 0.5 & 0.9 & 0.4 & 0.2 & 5.6 & 2. & 1.6 & 5.2 & 2.0 & 1.4 & 2.6 & 0.3 & 0.9 & 0.1 & 5.6 & 1.9 & 5.2 & 0.5 \\
\hline spms/ac15 & 2.7 & 0.4 & 0.4 & 0.8 & 0.4 & 0.2 & 5.4 & 1.9 & 1.8 & 5.4 & 1.7 & 1.2 & 2.7 & 0.2 & 0.8 & 0.1 & 5.4 & 1.8 & 5.4 & 0.4 \\
\hline
\end{tabular}

*Root length (RL); Shoot length (SL); Leaf area (LA); and Chlorophyll content (CC)

Expression of Spermine synthase (SPMS) and Thermospermine synthase (ACL5):

To investigate the normal expression pattern of spermine synthase (Spms) and thermospermine synthase (Acl5) genes, transgenic plants namely Spms promoter::GUS and Acl5 promoter:: GUS were grown on normal MS media for seven and fourteen days. After that the plants were carefully detached from the media and subjected to undergo GUS staining according to Sagor et al. (2011). Both the Spms and Acl5 promoter activity was detected in the cotyledons, true leaves and roots, but main difference is the strong induction of Acl5 in the vascular parts of the plants indicating some specific role in xylem differentiation and vascular development.

\section{Discussion}

Polyamines are positively charged aliphatic molecule with low molecular weight having two or more amino group which is found in all living organisms. The present study was planned to reveal the differential roles of tetraamine (Spm and T-Spm) in Arabidopsis thaliana using wild type (Col-0) and mutant lines (spms, acl5, spms/acl5) having different concentrations of spermine and thermospermine (Sagor et al., 2013). At first effect of exogenous spermine and thermospermine on wild type was examined under both normal and salt stress condition. T-Spm pretreated plants showed the highest root and shoot length compared to mock or Spm treated one. The chlorophyll content was also found to be higher in T-Spm pretreated plant. Similar results were also found by Knott et al., (2007), Minguet et al., (2008), Takano et al., (2012), where they showed that exogenous T-Spm can also act as hormone for the growth and development of plant via activating auxin or cytokinin signaling pathway gene and also hormonal deregulation. The wild type and both tetraamine deficient mutant (spms/acl5) or individual (either spms or acl5) mutants were grown on normal MS media or under stressful conditions but there was not much specific difference among wild type and mutant lines (spms, acl5 and spms/acl5) at seedling stages. Kakehi et al. (2008) showed acl5 mutant had dwarf phenotype at mature stage due to lack of T-Spm, but spms mutant had not such difference. In case of stress condition spms showed the highest tolerance than other wild and mutants through investigation of different parameter (root length, shoot length, leaf area and chlorophyll content). Yamaguchi et al. (2006, 2007) showed that tetraamine deficient mutant can compensate salt tolerance phenotype by exogenous application of Spm 
which was little bit different from our findings, but this may be due to some differential and tissue specific role of two different tetraamines; Spm and T-Spm. spms mutant cannot produce Spm but slightly higher T-Spm compared to normal plant (Sagor et al., 2011; Naka et al., 2009). This increase of T-Spm may have some crucial role in stress response. The expression pattern of tetraamine synthase genes were also studied using promoter::GUS transgenic approach. Both the SPMS and ACl5 gene differ from each other in terms of intensity and expression site. The intensity of ACL5 gene expression was found only in vascular system, whereas, Spms was throughout the leaf and stem including vascular part. Similar results were also obtained by Sagor, et al., (2011), Kakehi et al., (2008). They also found that ACL5 is highly involved in xylem differentiation and vascular development, whereas Spms is in stress responses (Clay and Nelson, 2005). All of the above discussion suggested that in Arabidopsis both the tetraamines have some specific and differential role based on their amount present either in soluble or conjugated form.

\section{Acknowledgements}

Dr. Tomonobu Kusano is acknowledged for providing the seeds of different Arabidopsis mutants. This work was supported by grants from Bangladesh Agricultural University Research System (BAURES) to GHMS (2017/262/BAU).

\section{References}

Alcázar, R., Marco, F., Cuevas, J. C., Patron, M., Ferrando, A., Carrasco, P., Tiburcio, T. and Altabella, A. F. 2006. Involvement of polyamines in plant response to abiotic stress. Biotechnol. Lett. 28: 1867-1876.

Bagni, N., Ruiz-Carrasco, K., Franceschetti, M., Fornalè, S., Fornasiero, R. B. and Tassoni, A. 2006. Polyamine metabolism and biosynthetic gene expression in Arabidopsis thaliana under salt stress. Plant Physiol. Biochem. 44: 776-786.

Clay, N. K. and Nelson, T. 2005. Arabidopsis thickvein mutation affects vein thickness and organ vascularization, and resides in a provascular cell-specific spermine synthase involved in vein definition and in polar auxin transport. Plant Physiol. 138: 767-777.

Fuell, C., Elliot, K. A., Hanfrey, C. C., Franceschetti, M. and Michael, A. J. 2010. Polyamine biosynthetic diversity in plants and algae. Plant Physiol. Biochem. 48:513-520.

Groppa, M. D., Benavides, M. P. and Tomaro, M. L. 2008. Polyamine metabolism in sunflower and wheat leaf discs under cadmium or copper stress. Plant Sci. 161: 481-488.

Hamana, K., Niitsu, M., Samejima, K. and Matsuzaki, S. 1991. Linear and branched pentaamines, hexaamines and heptaamines in seeds of Vicia sativa. Phytochem. 30: 3319-3322.

Hanfrey, C., Sommers, S., Mayer, M. J., Burtin, D. and Michael, A. J. 2001. Arabidopsis polyamine biosynthesis: absence of ornithine decarboxylase and the mechanism of arginine decarboxylase activity. Plant J. 27: 551-560.

Jefferson, R.A., Kavanagh, T.A. and Bevan, M.W. 1987. GUS fusions: $\beta$-glucuronidase as a sensitive and versatile gene fusion marker in higher plants. EMBO J. 6: 3901-3907.

Kakehi, J., Kuwashiro, Y., Niitsu, M. and Takahashi, T. 2008. Thermospermine is required for stem elongation in Arabidopsis thaliana. Plant Cell Physiol. 49: 1342-1349.

Kasukabe, Y., He, L., Nada, K., Misawa, S., Ihara, I. and Tachibana, S. 2004. Overexpression of spermidine synthase enhances tolerance to multiple environmental stresses and upregulates the expression of various stress-regulated genes in transgenic Arabidopsis thaliana. Plant Cell Physiol. 45: $712-722$.

Kitashiba, H., Hao, Y. J., Honda, C. and Moriguchi, T. 2005. Two types of spermine synthase genes, MdACL5 and MdSPMS are differentially involved in apple fruit development and cell growth. Gene. 3612: 101-111.

Knott, J. M., Römer, P. and Sumper, M. 2007. Putative spermine synthases from Thalassiosira pseudonana and Arabidopsis thaliana synthesize thermospermine rather than spermine. FEBS Lett. 581: 3081-3086.

Kusano, T., Berberich, T., Tateda, C. and Takahashi, Y. 2008. Polyamines: essential factors in growth and survival. Planta. 228: 367-381.

Kusano, T., Yamaguchi, K., Berberich, T. and Takahashi, Y. 2007. Advances in polyamine Research in 2007. J. Plant Res. 120: 345-350.

Minguet, E. G., Vera-Sirera, F., Marina, A., Carbonell, J. and Blázquez, M. A. 2008. Evolutionary diversification in polyamine biosynthesis. Mol. Biol. Evol. 25: 2119-2128.

Mitsuya, Y., Takahashi, Y., Berberich, T., Miyazaki, A., Matsumura, H., Takahashi, H., Terauchi, R. and Kusano, T. 2009. Spermine signaling plays a significant role in the defense response of Arabidopsis thaliana to cucumber mosaic virus. J. Plant Physiol. 166: 626-643.

Murashige, T. and Skoog, F. 1962. A revised medium for rapid growth and bioassays with tobacco tissue cultures. Physiol. Plant. 15: 473-497.

Naka, Y., Watanabe, K., Sagor, G. H. M., Niitsu, M., Pillai, M. A., Kusano, T. and Takahashi, Y. 2009. Quantitative analysis of polyamines including thermospermine during growth and salinity stress. Plant Phyisol. Biochem. (Polyamine special issue). 48: 527-533.

Niitsu, M. and Samejima, K. 1986. Synthesis of a series of linear pentaamines with three and four methylene chain intervals. Chem. Pharm Bull. (Tokyo). 34: 1032-1038.

Oshima, T. 1979. A new polyamine, thermospermine, 1, 12-diamino-4, 8-diazadodecane, from an extreme thermophile. J. Biol. Chem. 254: 8720-8722.

Sagor, G. H. M., Berberich, T., Takahashi, Y., Niitsu, M. and Kusano, T. 2013. The polyamine spermine protects Arabidopsis from heat stress-induced damage by increasing expression of heat shock-related genes. Trans. Res. 22 : (3), 595-605.

Sagor, G. H. M., Takahashi, H., Niitsu, M., Takahashi, Y., Berberich, T. and Kusano, T. 2012. Exogenous thermospermine has an activity to induce a subset of the defense genes and restrict cucumber mosaic virus multiplication in Arabidopsis thaliana. Plant Cell Rep. 31: (7), 1227-1232.

Sagor, G. H. M., Yamaguchi, K., Watanabe, K., Berberich, T., Kusano, T. and Takahashi, Y. 2011. Spatio-temporal expression analysis of Arabidopsis thaliana spermine synthase gene promoter. Plant Biotechnol. 28: 407-411.

Takahashi, Y., Berberich, T., Miyazaki, A., Seo, S., Ohashi, Y. and Kusano, T. 2003. Spermine signaling in tobacco: activation of mitogen-activated protein kinases by spermine is mediated through mitochondrial dysfunction. Plant J. 36: 820-829.

Takano, A., Kakehi, J. I. and Takahashi, T. 2012. Thermospermine is not a minor polyamine in the plant kingdom. Plant Cell Physiol. 53: 606-616.

Tiburcio, A. F., Altabella, T., Bitrián, M. and Alcázar, R. 2014. The roles of polyamines during the lifespan of plants: from development to stress. Planta. 240: 1-18.

Yamaguchi, K., Takahashi, Y., Berberich, T., Imai, A., Miyazaki, A., Takahashi, T., Michael, A. and Kusano, T. 2006. The polyamine spermine protects against high salt stress in Arabidopsis thaliana, FEBS Lett. 580: 783-6788.

Yamaguchi, K., Takahashi, Y., Berberich, T., Imai, A., Miyazaki, A., Takahashi, T. Michael, A. and Kusano, T. 2007. A protective role for the polyamine spermine against drought stress in Arabidopsis. Biochem. Biophys. Res. Commun. 352: $486-490$. 